\title{
Acute Pancreatitis with Colonic Pseudo-obstruction in a Patient with COVID-19
}

\author{
Jeremy Van ${ }^{a}$ Jason Kramer ${ }^{b}$ loannis Economou ${ }^{b}$ \\ aDepartment of Internal Medicine, Rush University Medical Center, Chicago, IL, USA; \\ ${ }^{b}$ Department of Gastroenterology, Rush University Medical Center, Chicago, IL, USA
}

Keywords

Acute pancreatitis $\cdot$ Intestinal obstruction $\cdot$ Abdominal pain

\begin{abstract}
Angiotensin-converting enzyme 2 receptor, the receptor used by severe acute respiratory syndrome coronavirus-2 (COVID-19) to infect cells, is found not only on respiratory epithelium but also in the small bowel, large bowel, and pancreas. There have been rare reports of acute pancreatitis (AP) in COVID-19 patients without an obvious etiology other than the underlying viral syndrome. We present a patient who was admitted with COVID-19 and developed AP and colonic pseudo-obstruction.
\end{abstract}

\section{Introduction}

In December 2019, severe acute respiratory syndrome coronavirus-2 (COVID-19) was first reported in Wuhan, China. Since then, there are over 124 million cases confirmed worldwide [1]. Common symptoms include fever, cough, myalgia, and shortness of breath [2]. Patients also have reported gastrointestinal symptoms. Around 66 of 254 people confirmed to have COVID-19, from December 2019 to February 2020 in China, were noted to have gastrointestinal symptoms such as nausea, vomiting, diarrhea, and abdominal pain [3]. Positive stool samples with COVID-19 have been found, supporting the possibility of fecal-oral transmission [4]. COVID-19 infects cells via angiotensin-converting enzyme 2 receptors (ACE2Rs) found not only on respiratory epithelium but also on gut enterocytes [5]. These receptors are also found on pancreatic islet 
cells and exocrine glands [6]. Infection of duodenal epithelium with further transmission to the pancreatic duct, acinar, and islet cells is a possible pathogenic mechanism [7]. Cases of acute pancreatitis (AP) have been rarely described in patients actively infected with COVID-19. We report a case of AP in a COVID-19 patient with abdominal pain, initially thought to be primarily from colonic pseudo-obstruction, which persisted after endoscopic colonic decompression.

\section{Case Report}

A 66-year-old male with a past medical history of pulmonary embolism, hypertension, type II diabetes mellitus, and chronic kidney disease presented to the hospital with a 3-day history of generalized weakness and shortness of breath. He had no significant past surgical or family history. His social history was remarkable for distant heroin use but no past smoking or alcohol history. Home medications included rivaroxaban, buprenorphine/naloxone, insulin glargine, and metoprolol. His vital signs on arrival included BP 147/79, HR 92, Temp $102.6^{\circ} \mathrm{F}$, RR 17, and Sp02 94\% on room air. Physical examination was remarkable for mild crackles in the bilateral lower lung fields. His WBC count was $1.18 \mathrm{~K} / \mu \mathrm{L}$. The HIV antigen and antibody were negative. His blood urea nitrogen and creatinine (Cr) were $40 \mathrm{mg} / \mathrm{dL}$ and $2.67 \mathrm{mg} / \mathrm{dL}$ (baseline $\mathrm{Cr} 2.0 \mathrm{mg} / \mathrm{dL}$ ). His liver function tests (LFTs) were within normal range. Urine drug screen and blood and urine cultures were negative. COVID-19 nasopharyngeal PCR was positive. His inflammatory markers were remarkable for C-reactive protein $43.7 \mathrm{mg} / \mathrm{L}$ and ferritin $931 \mathrm{ng} / \mathrm{mL}$. The chest radiograph demonstrated bilateral lower lobe airspace opacities. He was treated for COVID-19 with supportive management. On day 3 of hospitalization, he complained of epigastric abdominal pain and distension. An abdominal radiography (KUB) showed $9 \mathrm{~mm}$ dilated gas-filled large bowel (shown in Fig. 1). Of note, computed tomography (CT) abdomen/pelvis (AP) from 20 days prior did not show significant colonic dilation. Potassium, magnesium, and thyroid-stimulating hormone were within normal range. His LFTs were still in normal range. CT-AP without IV contrast demonstrated a diffusely dilated large bowel without a transition point (shown in Fig. 2); the gallbladder and pancreas were unremarkable. Tap water enemas and bisacodyl suppositories were administered with 2-3 bowel movements daily, but he continued to have abdominal pain. From day 6 to 8, repeat KUBs continued to have significant colonic dilatation with decreased frequency in bowel movements. IV erythromycin was trialed without improvement. Neostigmine therapy was not administered in the setting of significant renal impairment. On day 9, he was started on total parenteral nutrition
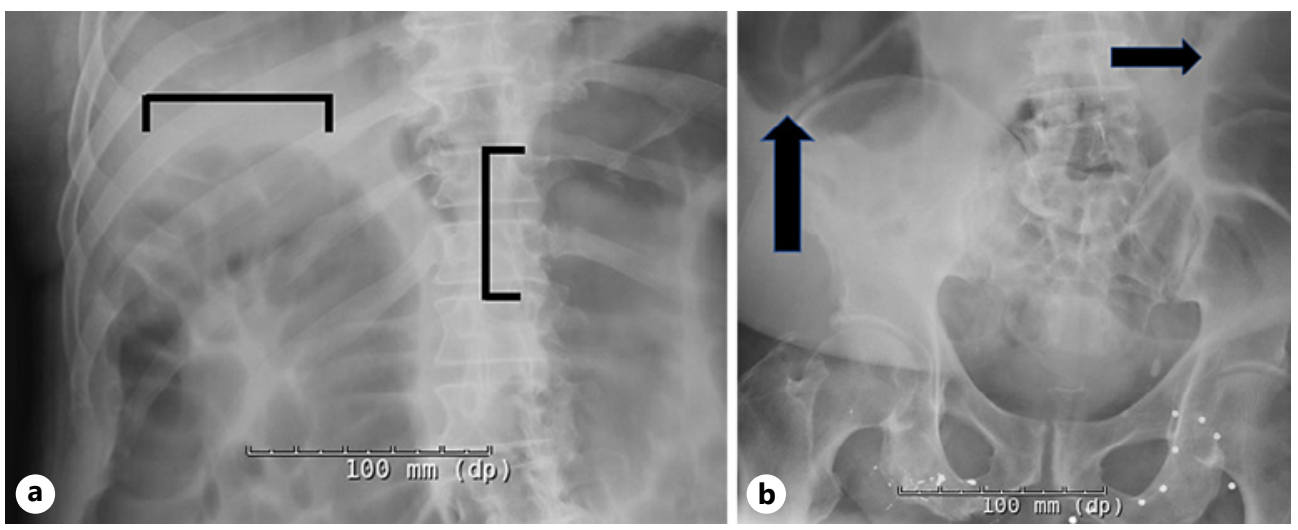

Fig. 1. a-b Abdominal radiograph (one view anterior-posterior) demonstrating a significantly dilated ( $9 \mathrm{~mm}$ diameter) gas-filled large bowel (black arrows and bars) concerning for colonic ileus. 

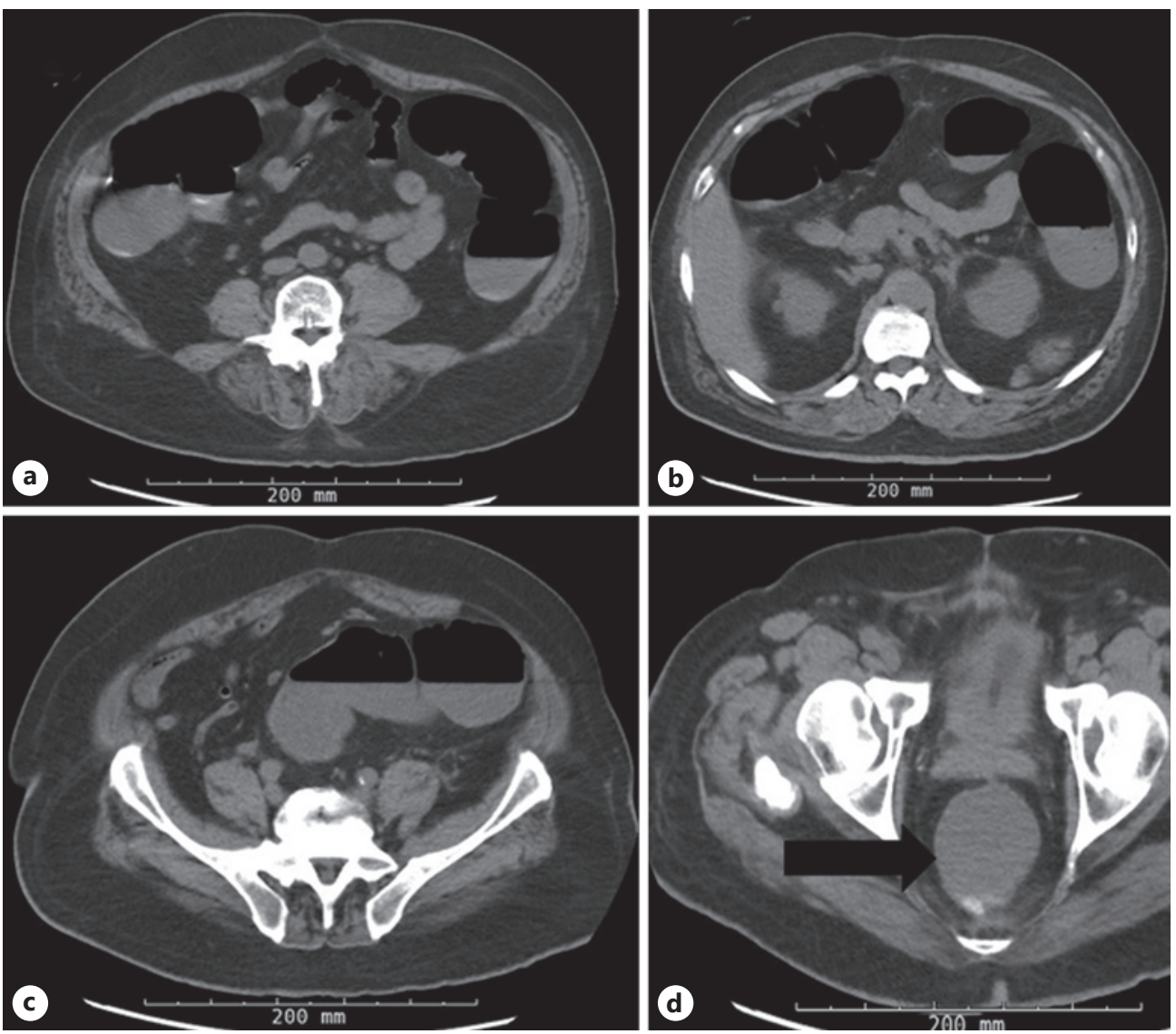

Fig. 2. a-c CT-AP without intravenous contrast (axial view) demonstrating a diffuse gas and fluid-filled dilated large bowel without definitive obstruction or transition point. d CT-AP without intravenous contrast (axial view) demonstrating a distended and fluid-filled rectum (black arrow).

with a repeat KUB demonstrating large bowel dilatation and cecal dilation greater than $10 \mathrm{~mm}$ (shown in Fig. 3a). Also, the patient had no BM for $>24 \mathrm{~h}$. Thus, an endoscopic colonic decompression was performed with improvement in dilation on KUB (shown in Fig. 3b). Postprocedure, he was less distended on abdominal examination, but he continued to have epigastric abdominal pain. Lipase and amylase were $959 \mathrm{U} / \mathrm{L}$ (normal range 0-52 U/L) and $425 \mathrm{U} / \mathrm{L}$ (normal range 25-115 U/L); LFTs were in normal range and stable, and $\mathrm{Cr}$, calcium, and triglycerides were $2.04 \mathrm{mg} / \mathrm{dL}, 8.7 \mathrm{mg} / \mathrm{dL}$, and $74 \mathrm{mg} / \mathrm{dL}$. On day 11 , the patient was hemodynamically stable, afebrile with only mild pain to palpation on his abdominal exam. His lipase downtrended from 959 to $739 \mathrm{U} / \mathrm{L}, \mathrm{C}$-reactive protein was stable at $38.4 \mathrm{mg} / \mathrm{L}$, and WBC had normalized from 1.18 to $8.83 \mathrm{~K} / \mu \mathrm{L}$. By day 15 , the patient continued to be hemodynamically stable with supportive management, was weaned off total parenteral nutrition, tolerated a soft diet, and had minimal abdominal pain. He was discharged to a skilled nursing facility on day 19.

\section{Discussion}

Acute pancreatitis has been described with viruses such as Coxsackie B and Epstein-Barr virus [8]. The pathogenesis for viral pancreatitis is unclear. Several proposed mechanisms include ampulla of Vater edema obstructing pancreatic fluid outflow, direct cytopathic pancreatic 

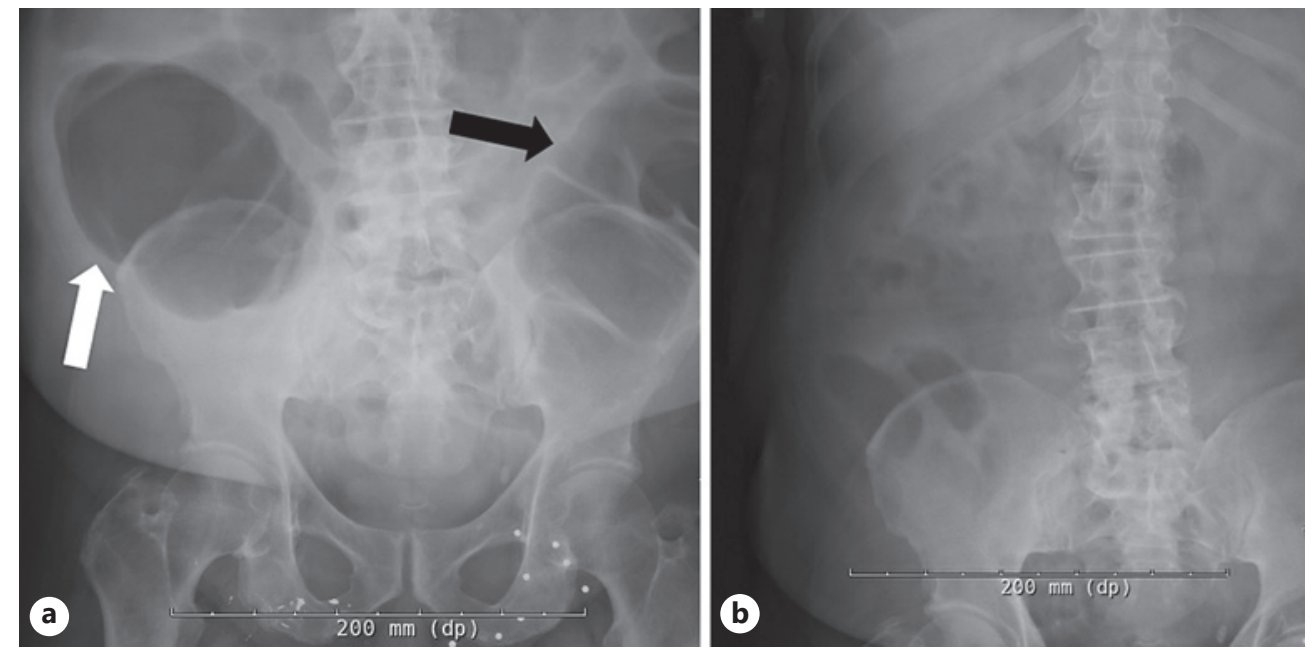

Fig. 3. a Abdominal radiograph (one view anterior-posterior, pre-endoscopic decompression) demonstrating multiple gas-filled dilated loops of large bowel (black arrow) and cecum (white arrow) greater than $10 \mathrm{~mm}$ dilation. b Abdominal radiograph (one view anterior-posterior, post-endoscopic decompression) demonstrating no dilation of large bowel.

injury, and indirect damage via systemic inflammatory and immune-mediated cellular responses [8, 9]. The latter two mechanisms have been proposed for COVID-19-induced AP [9]. A direct cytopathic mechanism is possible as there is ACE2R expression in small-bowel enterocytes and the pancreas [5]. Large bowel enterocytes also express ACE2R [10]. Our patient developed Ogilvie's syndrome, which has been described in a few COVID-19 patients [11]. As the patient concurrently had pancreatitis and Ogilvie syndrome, it is plausible both syndromes were impacted by COVID-19.

In a retrospective study evaluating COVID-19 versus non-COVID-19 patients with AP, there was $0.39 \%$ prevalence of AP among both groups. Of the COVID-19 patients, there was a $0.27 \%$ prevalence. Regardless of COVID-19 status, AP prevalence was low. But COVID-19 may still play a role in AP pathogenesis as 69\% of cases were "idiopathic" [12]. Although, in another similar retrospective study, comparison of AP frequency in COVID-19 versus non-COVID-19 patients resulted in an odds ratio of 0.41, questioning if COVID-19 has a role in pathogenesis. Also, interestingly, the rise in lipase and amylase was more moderate, and abdominal pain was less frequent in COVID-19 AP patients, making diagnosis in this patient population potentially difficult [13].

Per the revised Atlanta classification, AP diagnosis requires two out of three criteria: severe abdominal pain consistent with AP, serum lipase or amylase at least three times above the upper limit of normal, and imaging findings consistent with AP [14]. Our patient had severe epigastric abdominal pain and $18 \times$ and $4 \times$ the upper limit of normal of lipase and amylase, respectively. CT-AP was performed without IV contrast, limiting gallbladder and pancreas examination, as the patient had acute kidney injury. Although CT has a lower sensitivity of detecting gallstones compared to ultrasound, our patient never had elevated LFTs or jaundice [15] nor did he have an alcohol use history or hypertriglyceridemia. Our patient's etiology of AP was highly suspicious for COVID-19.

This case report highlights the importance of considering and evaluating for pancreatitis in COVID-19 patients presenting with abdominal pain. Further studies are needed to elucidate the potential relationship, pathogenesis, and incidence of AP in patients infected with COVID-19. 


\section{Acknowledgments}

We would like to thank the patient for allowing us to share their case.

\section{Statement of Ethics}

Written informed consent was obtained from the patient for publication of this case report and any corresponding images. This study protocol was reviewed, and the need for approval was waived by Rush IRB Administration at Rush University Medical Center.

\section{Conflict of Interest Statement}

The authors have no conflicts of interest to declare.

\section{Funding Sources}

This manuscript did not receive any funding.

\section{Author Contributions}

Dr. Jeremy Van, D0: Dr. Van's contributions included conception and design of the project, literature review, and writing the manuscript. Dr. Jason Kramer, MD: Dr. Kramer's contributions included conception and design of the project and review of the manuscript. Dr. Ioannis Economou: Dr. Economou's contributions included conception and design of the project, supervision, resources, and review of the manuscript. All the authors read and approved the final manuscript.

\section{Data Availability Statement}

All data generated or analyzed during this study are included in this article. Further inquiries can be directed to the corresponding author.

\section{References}

1 Johns Hopkins University of Medicine Coronavirus Resource Center Website. Available from: https://coronavirus. jhu.edu. Accessed 2021 Mar 24.

2 Huang C, Wang Y, Li X, Ren L, Zhao J, Hu Y, et al. Clinical features of patients infected with 2019 novel coronavirus in Wuhan, China. Lancet. 2020 Feb;395(10223):497-506.

3 Zhou Z, Zhao N, Shu Y, Han S, Chen B, Shu X. Effect of Gastrointestinal symptoms in patients with COVID-19. Gastroenterology. 2020 Jun;158(8):2294-7.

4 Holshue ML, DeBolt C, Lindquist S, Lofy KH, Wiesman J, Bruce H, et al. First case of 2019 novel coronavirus in the United States. N Engl J Med. 2020 Mar;382(10):929-36.

5 Lamers MM, Beumer J, van der Vaart J, Knoops K, Puschhof J, Breugem TI, et al. SARS-CoV-2 productively infects human gut enterocytes. Science. 2020 Jul;369(6499):50-4.

6 Bonney GK, Gao Y, Chew CA, Windsor JA. SARS-COV-2 associated acute pancreatitis: cause, consequence or epiphenomenon? Pancreatology. 2020 Jul;20(5):1017-8.

7 de-Madaria E, Capurso G. COVID-19 and acute pancreatitis: examining the causality. Nat Rev Gastroenterol Hepatol. 2021 Jan;18(1):3-4. 
8 Parenti DM, Steinberg W, Kang P. Infectious causes of acute pancreatitis. Pancreas. 1996 Nov;13(4):356-71.

9 Wang F, Wang H, Fan J, Zhang Y, Wang H, Zhao Q. Pancreatic injury patterns in patients with coronavirus disease 19 pneumonia. Gastroenterology. 2020 Jul;159(1):367-70.

10 Li MY, Li L, Zhang Y, Wang XS. Expression of the SARS-CoV-2 cell receptor gene ACE2 in a wide variety of human tissues. Infect Dis Poverty. 2020 Apr;9(1):45.

11 Kaafarani HMA, El Moheb M, Hwabejire JO, Naar L, Christensen MA, Breen K, et al. Gastrointestinal complications in critically ill patients with COVID-19. Ann Surg. 2020 Aug;272(2):61-2.

12 Inamdar S, Benias PC, Liu Y, Sejpal DV, Satapathy SK, Trindade AJ. Prevalence, risk factors, and outcomes of hospitalized patients with coronavirus disease 2019 presenting as acute pancreatitis. Gastroenterology. 2020 Dec;159(6):2226-e2.

13 Miro O, Llorens P, Jiménez S, Piñera P, Burillo-Putze G, Martín A, et al. A case-control emergency department-based analysis of acute pancreatitis in Covid-19: results of the UMC-19-S6. J Hepatobiliary Pancreat Sci. 2020 Dec.

14 Banks PA, Bollen TL, Dervenis C, Gooszen HG, Johnson CD, Sarr MG, et al. Classification of acute pancreatitis-2012: revision of the Atlanta classification and definitions by international consensus. Gut. 2013 Jan;62(1):102-11.

15 Memel D, Balfe D, Semelka R. The biliary tract. In: Lee J, Sagel S, Stanley R, editors. Computed body tomography with MRI correlation, Vol. 2. 3rd ed. Philadelphia, PA: Lippincott; 1998. p. 779-803. 